Original Article $\quad$ www.pjkd.com.pk

\title{
Causes of End Stage Kidney Disease in Maintenance Hemodialysis patients in District Swat, Khyber Pukhtonkhwa, Pakistan
}

\author{
Fawad Khalid, Asad ullah Khan, Adnan Fazal
}

Nawaz Sharif Kidney Hospital, Swat, Pakistan

Abstract:

Chronic kidney disease (CKD) affects $10-15 \%$ of the population worldwide and its prevalence is increasing.

Objective: To find the frequency of common diseases causing chronic kidney diseases (CKD) in dialysis dependent patients in District Swat, Khyber Pukhtonkhwa.

Methodology: Cross sectional study at Department of Nephrology Nawaz Sharif Kidney Hospital, District Swat, Khyber Pukhtonkhwa, Pakistan.

Results: Total of 110 patients were undergoing maintenance hemodialysis. There were 53(48.2\%) male and mean age was 54.40+16.32 years. Among 110 patients, only 9 (8.2\%) had dialysis once per week and 101(91.8\%) had dialysis twice per week hemodialysis. Majority, 64(58.2\%) patients were undergoing dialysis less than one year. 6(5.5\%) had hypertension, 33(30\%) had diabetes and 68(61.8\%) patients had both Diabetes and Hypertension. Out of 110, 39(35.5\%) patients were Hepatitis B positive, and 28(25.5\%) patients had Hepatitis C.

Conclusion:

Results of this study showed that the leading cause of chronic kidney disease (CKD) among dialysis patients was diabetes mellitus with or without hypertension and a high prevalence of both HBV and HCV.

Key words: Hemodialysis, frequency, diabetes mellitus, hypertension, HCV, HBV, HBsAg.

Corresponding Author:

Dr Fawad Khalid

Nawaz Sharif Kidney Hospital

Swat, Pakistan

Email: fawadkhalid004@gmail.com

PJKD 2020;5(2):54-59

\section{Introduction:}

Chronic kidney disease (CKD) affects $10-15 \%$ of the population worldwide and its prevalence is increasing. ${ }^{1,2}$ CKD ultimately results in end stage kidney disease (ESKD) over time requiring renal replacement therapy as dialysis or transplantation. Health care cost associated with ESKD is tremendous and strategies to reduce the burden of disease include identifying the underlying causes leading to ESKD with special attention to its pre-dialysis management.

Diabetes mellitus (DM) and hypertension are the two main causes of CKD in all high-income and middle-income countries, and also in many low-income countries. ${ }^{3,5,6}$ The prevalence of chroniclkidney disease (CKD) in the developed world is $13 \%$ and is recognized as a condition that elevates the risk of cardiovascular complications as well as kidney failure and other complications. ${ }^{7.8}$ Both hypertension 


\section{Causes of ESKD}

and chronic kidney disease (CKD) have been recognized as serious interconnected global public health issues.

According to a study from neighboring India, screening and early evaluation of kidney disease (SEEK) study, the overall prevalence of CKD stands at 17.2\%; and, approximately 6\% for CKD stage 3 and beyond. ${ }^{10}$ Hypertension and diabetes were the leading causes of CKD in this study. In Pakistan, although no large study exists, however local data probably is not different than this.

It is not surprising therefore that hypertension and DM are the two main leading causes of ESKD globally. ${ }^{7}$ Studies including maintenance hemodialysis (MHD) patients from Pakistan show a similar picture with high DM as the leading cause of ESKD. ${ }^{6}$

The rationale of the study was to find the frequency of common underlying diseases causing chronic kidney diseases (CKD) in our maintenance hemodialysis (MHD) patients in District Swat, Khyber Pukhtonkhwa, Pakistan since no study exists from this region.

\section{Methodology:}

This descriptive cross-sectional study was conducted from January 2021 to $31^{\text {st }}$ March in the Nephrology department of Nawaz Sharif Kidney Hospital, Swat. Since no formal institutional review board existed, the study was approved by the administration for any ethical concerns and approved. Informed written consent was obtained from patients about the data collection and privacy. All patients undergoing MHD were included in this frequency analysis.

None of the patients refused to complete the interview. The data was collected for age, gender, duration of hemodialysis, causes of underlying kidney disease, Hepatitis $\mathrm{B}$ and $\mathrm{C}$ status and vaccination for HBV.

Data was entered in SPSS 21 (IBM, Armonk, New York, USA). Age, blood pressure, serum creatinine, urea was presented as mean and standard deviation. Categorical data like gender, hepatitis B and C status were presented as frequencies \& percentage.

\section{Results:}

In our study, total 110 patients were included. Patient characteristics are described in table 1. Majority were females $57(51.8 \%)$ and mean age was $54.40 \pm 16.32$ years. Majority of the patients $42(38.2 \%)$ were between the 56-70 years of age group and 24(21.8\%) patients between the 41-55 years of age group, Table: 1.

Other patient characteristics as height, weight, systolic and Diastolic blood pressure, blood sugar level, serum creatinine and urea level are shown in Table: 2.

Among 110 patients, only 9 (8.2\%) had dialysis once per week and majority, 101(91.8\%) had dialysis two times per week. The mean duration of dialysis was15.07 17.16 months. Majority of patients, 64 (58.2\%) were undergoing MHD for less than one year, Table: 3. 


\section{Causes of ESKD}

Diabetes Mellitus was the commonest etiology for ESKD, 33(30\%) had diabetes and 68(61.8\%) patients had Diabetes and Hypertension, Table: 3.

Among our MHD patients, HBV was common at a frequency of 39(35.5\%) and 28(25.5\%) patients had hepatitis C, Table: 4.

Table 1: Frequency distribution of Gender and Age of 110 maintenance hemodialysis patients.

\begin{tabular}{|c|c|c|}
\hline \multicolumn{2}{|c|}{} & Frequency (\%) \\
\hline \multirow{3}{*}{ Gender } & Male & $53(48.2 \%)$ \\
\cline { 2 - 3 } & Female & $57(51.8 \%)$ \\
\hline \multirow{4}{*}{ Age (Years) } & Mean+ SD & $54.40 \pm 16.31$ \\
\cline { 2 - 3 } & $\mathbf{1 0 - 2 5 y e a r s}$ & $6(5.5 \%)$ \\
\cline { 2 - 3 } & $\mathbf{2 6 - 4 0 y e a r s}$ & $18(16.4 \%)$ \\
\cline { 2 - 3 } & $\mathbf{4 1 - 5 5 y e a r s}$ & $24(21.8 \%)$ \\
\cline { 2 - 3 } & $\mathbf{5 6 - 7 0 y e a r s}$ & $42(38.2 \%)$ \\
\cline { 2 - 3 } & $\mathbf{7 1 - 8 5 y e a r s}$ & $20(18.2 \%)$ \\
\hline
\end{tabular}

Table 2: Descriptive Statistics of of 110 maintenance hemodialysis patient for Height, Weight, Blood Pressure, sugar Level, Serum Creatinine \&Urea

\begin{tabular}{|l|c|}
\hline & Mean (SD) \\
\hline Weight Kg & $80.69 \pm 14.94$ \\
\hline Systolic Blood Pressure mm of Hg & $145.18 \pm 17.38$ \\
\hline Diastolic Blood Pressure mm of Hg & $92.31 \pm 8.25$ \\
\hline Blood Sugar Level mg/dl & $311.7 \pm 98.166$ \\
\hline Serum Creatinine mg/dl & $6.20 \pm 2.60$ \\
\hline Serum Urea mg/dl & $152.15 \pm 58.27$ \\
\hline
\end{tabular}

\section{Discussion:}

Our study is the first from this part of Pakistan detailing the causes of ESKD. It highlights the presence of DM as the major underlying cause of ESKD similar to other parts of Pakistan and globally. ${ }^{6,11-14}$

Pakistan is one of the countries where incidence of DM is fast increasing and over the time its complication including diabetic nephropathy and ESKD will increase. ${ }^{15}$ Our study supports this notion and highlights the necessity to address health education. dietary and lifestyle modifications on emergency basis. In fact prevention and proper management of diabetic patients is the only solution to decrease the burden of ESKD. ${ }^{16}$ Diabetes was present in majority of our patients, 101(91.8\%), and among them almost $60 \%$ had hypertension also. In fact studies from pakistan repeatedly show the same pattern of eitiology of ESKD from different areas of Pakistan. ${ }^{6,11-14}$ 


\section{Causes of ESKD}

Table 3: Frequency Distribution of Duration of dialysis of 110 maintenance hemodialysis patients.

\begin{tabular}{|l|c|}
\hline Duration of Dialysis & Frequency (\%) \\
\hline Mean + SD & $15.07 \pm 17.16$ \\
\hline Less than one year & $64(58.2 \%)$ \\
\hline $\mathbf{1 - 3}$ years & $36(32.7 \%)$ \\
\hline$>\mathbf{3}$ years & $10(9.1 \%)$ \\
\hline
\end{tabular}

Table 4: Frequency Distribution of Causes of Disease of 110 maintenance hemodialysis patients.

\begin{tabular}{|l|c|}
\hline Causes of Disease & Frequency (\%) \\
\hline Alport Syndrome & $1(0.9 \%)$ \\
\hline Diabetes Mellitus (DM) & $33(30 \%)$ \\
\hline Hypertension (HTN) & $6(5.5 \%)$ \\
\hline DM+HTN & $68(61.8 \%)$ \\
\hline DM+HTN+HF & $2(1.8 \%)$ \\
\hline
\end{tabular}

Health education therefore at all levels and provision of facilities such as parks etc for exercise is an essntial component of management strategies. Unfortunatley majority of the data of CKD in Pakistan is still from the MHD population and paucity of data among pre-ESKD patients is evident as no local studies looking at long term follow up of CKD patietns are available. We as health care providers, researchers should look into this population and try to identify the local dynamics of the disease and its management. The dialysis units all over Paksitan including ours have long waiting lists of dialysis patients and by proper management of CKD pre-dialysis this list can be shortened." ${ }^{5,14}$

CKD mangement itself and MHD for ESKD over a period of time leads to a huge fianancial burden for the patient and family since the coverage for costly hemodialysis and medication is availble on payment in most areas of Pakistan. ${ }^{5,14}$ Nevertheless, a large number of patietns are being treated free of cost in goverenmental and charity based dialysis units in Pakistan. Many of such patietns still may have to buy medications and pay for procedures and hospitaliztions frequently. Our dialysis unit is a govermnment based hospital and provides free of cost dialysis and medications. Recently introduction of insurance health card scheme has improved coverage for the hemodialysis sessions, however it doesnot still cover for the pre ESKD stage.

Thrice weekly sessions of hemodialysis is the recommended norm internationally, however none of our patietns were on thrice weekly dialysis as practice in the low or middle income countries. ${ }^{17,18}$ In fact, 9(8.2\%) were undergoing MHD only one time per week and rest twice per week. The reason for twice weekly is mainly due to financial constraints limited availability of dialysis beds with long waiting lists. 


\section{Causes of ESKD}

Nevertheless, recent studies do favorably argue that twice weekly dialysis is adequate and may preserve residual renal function as weel as overall quality of life. ${ }^{17}$

Another important finding of our study is the very high prevalence of HBV and HCV among our MHD patients. ${ }^{18}$ Data from Pakistan does suggest a decreasing trend of $\mathrm{HBV}$ due to $\mathrm{HBV}$ vaccination and thus argues for an urgent review of the HBV vaccination practices among our incident dialysis and CKD patients in Swat since only 64.5\% patients were vaccinated for HBV. ${ }^{6,2021}$ Similarly, HCV status of our patients was similarly high but at the same level reported in the literature from Pakistan. ${ }^{6}$ We hope that with the newer antiviral medications, there will be a decreasing trend of HCV seroconversion and prealence.

Table 5: Frequency Distribution of Hepatitis B \&C of 110 maintenance hemodialysis patients.

\begin{tabular}{|l|c|c|}
\hline \multirow{2}{*}{} & \multicolumn{2}{|c|}{ Frequency (\%) } \\
\cline { 2 - 3 } & Yes & No \\
\hline Hepatitis B & $39(35.5 \%)$ & $71(64.5 \%)$ \\
\hline Hepatitis C & $28(25.5 \%)$ & $82(74.5 \%)$ \\
\hline Hep B Vaccinated & $71(64.5 \%)$ & $39(35.5 \%)$ \\
\hline
\end{tabular}

\section{Conclusion:}

The results of this study showed that the leading cause of CKD among our dialysis patients was DM and hypertension. We suggest that preventive strategies to control diabetes and hypertension will ultimately result in decreasing the numbers of ESKD patients and health care costs.

Other important finding of our study is the high prevalence of HBV among our MHD patients and suboptimal percentage of patients having been vaccinated. It is imperative that urgent attention to HBV vaccination in general population and at-risk patients including CKD patients is needed.

Disclosure: None Declared

Acknowledgement: We would like to thank all the dialysis technicians and supportive staff of the hemodialysis unit for their support in preparing the manuscript.

\section{References:}

1. Webster AC, Nagler EV, Morton RL, Masson P. Chronic kidney disease. The lancet. 2017;389(10075):1238-52

2. Mills KT, Xu Y, Zhang W, Bundy JD, Chen C-S, Kelly TN, et al. A systematic analysis of worldwide population-based data on the global burden of chronic kidney disease in 2010. Kidney international. 2015;88(5):950-7.

3. Thurlow JS, Joshi M, Yan G, Norris KC, Agodoa LY, Yuan CM, et.al. Global epidemiology of end-stage kidney disease and disparities in kidney replacement therapy. Am J Nephrol 2021;52:98-107

4. Hall ME, do Carmo JM, da Silva AA, Juncos LA, Wang Z, Hall JE. Obesity, hypertension, and chronic kidney disease. Int J Nephrol Renovasc Dis. 2014;7:75-88

5. Imtiaz S, Alam A. Is hemodialysis the most feasible dialysis modality for Pakistan? JPMA 2021;71(2):528-30.

6. Shahbaz M. Data from a large hemodialysis center form an industrial city: demographics, etiology and hepatitis status. Pak J Kidney Dis 2019;(10):173-79. 


\section{Causes of ESKD}

7. Stevens PE, Levin A. Evaluation and management of chronic kidney disease: synopsis of the kidney disease: improving global outcomes 2012 clinical practice guideline. Annals of internal medicine. 2013;158(11):825-30

8. Coresh J, Selvin E, Stevens LA, Manzi J, Kusek JW, Eggers P, et al. Prevalence of chronic kidney disease in the United States. Jama. 2007;298(17):2038-47

9. Jha V, Garcia-Garcia G, Iseki K, Li Z, Naicker S, Plattner B, et al. Chronic kidney disease: global dimension and perspectives. The Lancet. 2013;382(9888):260-72

10. Singh AK, Farag YM, Mittal BV, Subramanian KK, Reddy SRK, Acharya VN, et al. Epidemiology and risk factors of chronic kidney disease in India - results from the SEEK (screening and early evaluation of kidney disease) study. BMC nephrology. 2013;14(1):1-10

11. Ali M, Ejaz A, Iram H, Solangi SA, Junejo AM, Solangi SA. Frequency of intradialytic complications in patients of end-stage renal disease on maintenance hemodialysis. Cureus 13(1): e12641.

12. Anwar S, Zainab A, Mazhar S. Lipid abnormalities using random sampling inpatients with end stage renal disease on thrice weekly hemodialysis. Pak J Kidney Dis. 2020;4(4):328-31.

13. Imtiaz M, Anees M, Gul S. Epidemiological profile of patients with end stage renal disease on maintenance hemodialysis in tertiary referral hospitals. Pak J Kidney Dis 2019;(10):30-34.

14. Irshad AN, Rafique Z, Rehman HS, Asrar A. Socioeconomic status of maintenance hemodialysis patients from a tertiary care hospital in Lahore. Pak J Kidney Dis 2019;(10):180-186.

15. Akhtar S, Nasir JA, Abbas T, Aqsa Sarwar A. Diabetes in Pakistan: A systematic review and meta-analysis. Pak J Med Sci 2019;35(4):1173-1178.

16. Bello AK, Levin A, Manns BJ, Feehally J, Drueke T, Faruque L et al. Effective CKD care in European countries: challenges and opportunities for health policy. American Journal of Kidney Diseases. 2015;65(1):15-25

17. Meyer TW, Hostetter TH, Watnick S. Twice-weekly hemodialysis is an option for many patients in times of dialysis unit stress. J Am Soc Nephrol. 2020;31:1141-42.

18. Savla D, Chertow GM, Timothy Meyer M, Anand S.Can twice weekly hemodialysis expand patient access under resource constraints? Hemodial Int 2017;21(4): 445-52

19. Winston A, Wurcel AG, Gordon C, Goyal N. Viral hepatitis in patients on hemodialysis. Seminars in Dialysis. 2020;33(3):254-262.

20. Asif M, Raheel Ahmed, Ahmed T, Zia Uddin, Zahid M, Ullah I . Frequency of hepatitis-B and C in patients on haemodialysis for end stage renal disease in tertiary care hospitals: a multicentre study. J Ayub Med Coll Abbottabad 2020;32(3)

21. Tarif N, Riaz MM, Sabir O, Akhter R, Rafique K, Rizvi N. Prevalence of hepatitis B core antibodies with negative hepatitis B surface antigen in dialysis and chronic kidney disease patients. Saudi J Kidney Dis Transpl 2017;28(4):869-873 